\title{
How early maternal separation and juvenile experience with pups affect maternal behavior and emotionality in adult postpartum rats
}

\author{
STEPHANIE L. REES and ALISON S. FLEMING \\ University of Toronto, Mississauga, Ontario, Canada
}

\begin{abstract}
To assess the effects of preweaning and juvenile experiences on adult maternal behavior, two experiments were completed. In Experiment 1, pups were separated for long periods or short periods or were left undisturbed over the 1st week. Following weaning, the rats were exposed to foster pups over a 5-day period or were left undisturbed. There were no effects of early experience on juvenile behavior. In adult postpartum maternal tests, short separations reduced latencies to express maternal behaviors, whereas long separations combined with juvenile exposure to pups increased latencies. In Experiment 2, pups were separated for long periods or were left undisturbed over the 1st week. Following weaning, the rats were exposed to foster pups for 4 days or 8 days or were left undisturbed. Long separation retarded maternal responding in juvenile animals but had no effect on the adults. Four days of juvenile experience had negative effects on adult behavior, whereas 8 days did not have an effect.
\end{abstract}

The purpose of these experiments was to determine whether maternal deprivation of the rat pup, during the 1 st week of life, affects the development of maternal behavior and its postpartum expression in adulthood. A second purpose was to determine whether deficits in adult maternal behavior, produced by early maternal deprivation, could be reversed by experience with pups during the juvenile period.

The somatosensory and chemosensory stimulation received by pups from their mothers throughout the preweaning period is important for the pups' normal development (Fleming, O'Day, \& Kraemer, 1999; Wilson \& Sullivan, 1994). When the interaction between pups and their mothers is disrupted, future behaviors can be influenced also. Maternal deprivation leads either to a "positive," more robust behavioral outcome or to deficits in behavior, depending on the length and conditions of the separation. Short periods of deprivation of the offspring from their mother, known as the handling paradigm, can produce beneficial effects that result in improved response to stressful situations, reduced emotionality, enhanced learning, and better maternal responsiveness in adulthood (Caldji, Francis, Sharma, Plotsky, \& Meaney, 2000; Dorner, Plaschke, Tonjes, \& Wenzel, 1984; Francis, Diorio, Liu, \& Meaney, 1999; Liu et al., 1997). These benefits occur not through being deprived of maternal care for brief periods of time, but rather through enhanced, intensive maternal care-specifically, increased lickingwhen the pups are returned to the nest. Conversely, ma-

This research was supported by NSERC to A.S.F. Correspondence concerning this article should be addressed to A. S. Fleming, Department of Psychology, University of Toronto at Mississauga, 3359 Mississauga Road North, Mississauga, ON, L5L 1C6 Canada (e-mail: fleming@psych.utoronto.ca). nipulations that result in a reduction in licking by mothers-for example, through olfactory bulbectomy of the mother, marking the offspring with a novel odorant, or an increase in litter size-also result in offspring that lick their own pups less when they are tested in adulthood (Fleming et al., 2001). Extended periods of separation, which can range from 1 to $24 \mathrm{~h}$ daily, are also deleterious in their effects on adult maternal behavior. Prolonged separation can lead to a more prolonged stress response, higher emotionality, poorer attention, poorer learning, and more erratic adult maternal behavior (Hall, 1998; Kuhn \& Schanberg, 1998).

These effects may result from the reduced maternal care, such as the reduction in the amount of licking received, from the nutritional loss by pups during their removal from the nest or, possibly, from the physiological changes that are associated with being separated from mother, litter, and nest and placed into a novel environment that lacks the familiar temperature, somatosensory, and olfactory cues. Long periods of deprivation over an extended time obviously disrupt infant-mother interactions, since bouts of nursing, licking, feeding, and retrieving will be altered. Five hours of daily deprivation from the mother over the first 18 days of life result in offspring that show reduced licking of and crouching over their own pups (Lovic, Gonzalez, \& Fleming, 2001). Total maternal deprivation, or development in isolation through artificial rearing, has even more pronounced effects, with deficits being found in every aspect of maternal behavior (A. Gonzalez, Lovic, Ward, Wainwright, \& Fleming, 2001).

In addition to producing changes in adult maternal behavior, preweaning isolation of pups also produces changes in "emotional" behavior; depending on the extent of the deprivation, on the emotionality test administered, and on the interpretation of the behavioral endpoints, early de- 
privation produces increased (Caldji et al., 2000) or no (Campbell \& Spear, 1999; Lehmann, Pryce, Bettschen, \& Feldon, 1999; Lovic et al., 2001) change in "fear" and "emotionality," as assessed by adult open-field activity and plus-maze performance.

Despite these problems of interpretation, there is a substantial literature showing that early deprivation from mothers and the nest have long-term effects on the development of the animals' hypothalamic-pituitary-adrenal (HPA) system and responses to stressors (Anisman, Zaharia, Meaney, \& Merali, 1998; Plotsky \& Meaney, 1993). Whether this elevated HPA system mediates these alterations in adult emotional behavior in general and in maternal behavior more specifically is not known. However, there is now a substantial literature documenting that the new mother rat who is responsive to her offspring is also less neophobic in a novel environment and is more attracted to pup-related cues that are novel (Fleming, Cheung, Myhal, \& Kessler, 1989; Fleming \& Luebke, 1981). These data indicate that one of the mechanisms by which early maternal deprivation could affect adult maternal behavior is by altering the "fear" system. In the study we, therefore, examined the relationship between the effects of maternal deprivation on adult fear and maternal behavior in the same animals.

Juvenile animals, when exposed to neonatal pups, will also demonstrate maternal behaviors (Gray \& Chesley, 1984). Although displaying many of the maternal behaviors, juveniles do not consistently display the fully integrated pattern of adultlike maternal behavior (Brunelli, Shindledecker, \& Hofer, 1985). Instead, the behavior toward foster pups is intermixed with play behaviors (Brunelli et al., 1985), although the play and maternal behaviors can be differentiated from each other by the differences shown by juveniles in their responses to pups versus toys (Gray \& Chesley, 1984). Potentially, the enhancement of adult maternal behavior through experience with pups as a juvenile may be dependent on whether full maternal behavior - that is, retrieval of foster pups-is displayed during the juvenile experience. Interestingly, virgin animals with undisturbed neonatal experiences that have had previous juvenile exposure to pups are more maternally responsive to foster pups and exhibit a shorter latency to the onset of maternal behavior following maternal induction procedures in which rats are exposed to foster pups until maternal behavior toward these pups is shown (Gray \& Chesley, 1984; Kinsley \& Bridges, 1988).

What is not known is how early maternal deprivation in combination with juvenile experience affects adult maternal behavior. Complete isolation from a mother during the first few days of life can influence pup-directed behaviors both in the juvenile period and in adulthood (A. Gonzalez \& Fleming, 2001). Partial maternal deprivation, however, does not seem to affect juvenile responsiveness to pups, although it does have a limited effect on adult maternal behavior (Lovic et al., 2001). Potentially, because deprived animals become poorer parents than do nondeprived animals (Lovic et al., 2001) and juvenile exposure to pups improves adult maternal behavior (Gray \& Chesley, 1984; Kinsley \& Bridges, 1988), juvenile exposure to foster pups may compensate for some of the deficits caused by deprivation and may improve maternal care in deprived animals.

To assess the effects of early deprivation and of juvenile nonmaternal exposure to pups and their interaction on adult behaviors, in the first experiment, female rats received one of three early experience manipulations over the 1st week of life: short separation (15 min of being separated from the mother), long separation ( $3 \mathrm{~h}$ of being separated from the mother and peers), and being left undisturbed. The animals then either were given a 5-day exposure to foster pups or were left undisturbed during the juvenile period and were tested during adulthood for emotional behaviors and for postpartum maternal behavior. A second experiment was undertaken to further explore the effects of juvenile experience with pupsespecially, maternal experience-and its interactive effects with early deprivations on adult behavior. In this study, animals in the 3-h separated and undisturbed conditions were given 4 days, 8 days, or no experience with pups during the juvenile period and then were tested for postpartum maternal behavior in adulthood.

\section{EXPERIMENT 1}

\section{Method}

\section{Subjects and Treatment}

The subjects were 43 female Sprague-Dawley rats born at the University of Toronto at Mississauga, from a stock originally obtained from Charles River Farms in Quebec. The subjects were maintained on a 12:12-h light:dark cycle, with lights on at $0800 \mathrm{~h}$. Room temperature and humidity were maintained at $24.0^{\circ} \mathrm{C}$ and $40 \%$, respectively. The treatment groups included the following: short separation/juvenile experience (SS/JE; $n=8)$, short separation/ no juvenile experience (SS/NJE; $n=6$ ), long separation/juvenile experience (LS/JE; $n=7$ ), long separation/no juvenile experience (LS/NJE; $n=9$ ), undisturbed/juvenile experience (U/JE; $n=6$ ), and undisturbed /no juvenile experience (U/NJE; $n=7$ ).

The subjects were separated or left undisturbed from Postnatal Days (PND) 2 to 9. On each day, short separation consisted of removing the mother from the cage, then removing the pups from the cage and placing the entire litter into a plastic container lined with clean bedding for $15 \mathrm{~min}$. The plastic container was maintained at nest temperature (approximately $34^{\circ} \mathrm{C}$ ) through the use of heating pads placed underneath the containers. The pups were returned to the nest after the 15-min period; then the mother was also returned to the nest. Short separation was used as a control for the effects of handling that occurred during separation.

Long separation consisted of the same procedure as short separation, except that the pups were removed for a period of $180 \mathrm{~min}$ and were isolated from each other in separate plastic containers lined with bedding. The undisturbed subjects were left in their home cage, and the mother was not removed. To control for potential litter effects, an entire litter received each treatment. Litters were culled to contain 3 females and 3 males, but only 2 female pups from each litter were kept for further testing. One female from each litter was assigned to each of the juvenile conditions.

\section{Maternal Behavior Test During the Juvenile Period}

On PND 21, the pups were weaned from their mothers and placed in separate observation cages with nesting material. From PNDs 22 to 26, half of the subjects from each of the treatment groups were observed with six 2- to 5-day-old pups, whereas the other half were 
left undisturbed. For each day during this period, the pups were placed in the observation cage in the corner opposite the subject. Every $5 \mathrm{sec}$ over the following $8 \mathrm{~min}$, the behavior of the animal was recorded. Prior to these testing sessions, the positions of the subject, the pups, and the nesting material were noted. The quality of the nest was also noted.

The maternal behaviors were recorded as follows: (1) retrieving, when the subject retrieved a pup to the nest area; (2) body licking, when the subject licked anywhere on the body of a pup; (3) genital licking, when the subject licked the anogenital region of a pup; (4) lying in contact, when the subject was lying down and touching the pups; (5) nest building, when the subject used the provided nesting material to build a nest; (6) sniff ing pups, when the subject sniffed the pups; (7) mouthing/pup pick-up, when the subject used her mouth to pick up the pup. The nonmaternal behaviors were recorded as follows: (1) sniffing air, when the subject sniffed the air; (2) grooming, when the subject self-groomed.

Foster pups were left in the animal's home cage with the subject for the duration of the testing period (PNDs 22-26); however, the pups were replaced with a new set of pups every $24 \mathrm{~h}$ at the beginning of each 8-min testing session. Two spot checks were also taken at 2 and $4 \mathrm{~h}$ following each testing session. For these spot checks, the positions of the subject, the pups, and the nesting material were noted. The subjects were separated and placed in normal cages upon completion of this juvenile maternal testing.

\section{Maternal Behavior Test During Adulthood}

The subjects were habituated to a room with a reversed light cycle. After a week of habituation, each female subject was placed with a male rat for a period of 5 days to ensure impregnation. Six days after the 5th day of mating, the subjects were switched back to their original light cycle to ensure that the subjects would give birth during the day (Mayer \& Rosenblatt, 1998). During parturition, the pups were removed from the subjects at 15-min intervals until the entire litter was born. For maternal behavior observations, 2- to 5 -day-old foster pups were placed in the cage with subjects 4 days after parturition, so that parturitional hormones would no longer be influential. If an animal was not pregnant, it was returned to the reversed cycle to be rehabituated and remated a second time. If this did not result in pregnancy, the animals were not tested any further. Only 1 animal did not become pregnant after two pairings with a male.

For the maternal behavior observations, the subjects were placed in large, clear cages and were given six donor pups. The same procedure used to observe maternal behavior over a 5-day period during the juvenile period was used for the adult observations, except that the latter observations were recorded with an event recorder that provided a continuous record of all ongoing behavior and permitted analyses of frequency and duration of behavior. In addition to the behaviors recorded for the juvenile maternal observations, hovering (crouching over foster pups), lactating posture (when the subject positioned herself over the pups and arched her back), and general licking (licking and genital licking) were recorded. The latency to become maternal was defined as the number of days until retrieval of all 6 pups to the nest during the 8-min testing period on 2 successive days of testing. Maternal behavioral testing was terminated for postpartum animals after 5 days of testing. Only 4 animals did not respond maternally as adults.

\section{Emotionality Tests: Emergence Test and Open-Field Test}

On PND 58, the day before mating, each subject was tested in the emergence test and the open field. The subject was placed in the start box $(21 \times 21.5 \times 20 \mathrm{~cm})$ for a 5 -min habituation period, after which the door separating the start box and the open field was opened. The latency to enter the open-f ield box $(150 \times 150 \times$ $50 \mathrm{~cm}$ ) was recorded, with a ceiling of $10 \mathrm{~min}$, after which the animals were gently pushed into the open field. All four paws had to be in the open field for a completed emergence. The number of times the animal puts its nose and head through the opening was also recorded.

The open-field test occurred over a 5-min period, which began once the subject entered the open field from the start box. While in this arena, the subject's movements were monitored by recording the number of square crossings per minute, as defined by 100 equal squares marked in the open field. The number of square crossings and entries into peripheral (outer) and central (inner) squares was recorded, as well as the number of times the subject reared, groomed, urinated, and defecated.

\section{Results}

To simplify the presentation of the following results, effects relating to either body licking or genital licking are referred to simply as licking; effects relating to hovering over pups or lactating posture are designated as crouch. Also, note that nonparametric tests were done where appropriate, but similar patterns of results were found as in the parametric tests, so only the parametric tests are reported throughout this paper.

\section{Juvenile Maternal Behaviors}

Percentage of juvenile animals showing maternal behaviors. In terms of pup-oriented behaviors, by the 3 rd test day, over $50 \%$ of the animals in each of the early experience groups had adopted a hover/crouch posture over young and also had demonstrated licking of pups. By the last (5th) day of testing, virtually all the animals in all the groups had exhibited these behaviors and spent considerable portions of the test period crouching over the pups. Despite the close proximal contact, only $30 \%-$ $40 \%$ of the animals in any group showed maternal-like licking, and only 10\%-30\% showed pup retrievals during the 5-day test period (Table 1). The groups did not differ in the percentage of animals showing the different maternal behaviors during the juvenile period.

Duration of behaviors across the test period. To determine the effects of early experience on juvenile maternal behavior, a series of 3 (early experience) $\times 5$ (days) repeated measures analyses of variance (ANOVA) was computed for the total time of different behaviors shown during the juvenile period. The results indicated that there were no significant main effects of early experience for total time spent in any of the behaviors by juvenile animals. There was a main effect of days for the duration of some behaviors-specifically, for licking of foster pups $[F(4,72)=6.968, p<.001]$ and lying in contact with foster pups $[F(4,72)=5.956, p<.002]$. More licking and lying in contact occurred during the last few days of the observation period than during the first few days. Toward the

Table 1

Percentage of Juvenile Animals Showing Maternal Behaviors in Experiment 1

\begin{tabular}{lccc}
\hline Behavior & Long-Separated & Short-Separated & Undisturbed \\
\hline Retrieve & 10 & 18.2 & 27.3 \\
Body lick & 90 & 100 & 100 \\
Genital lick & 10 & 9 & 36.4 \\
Hover & 100 & 100 & 100 \\
\hline
\end{tabular}


end of the observation period, an increasing number of animals in all the groups demonstrated components of maternal behavior. Other behaviors did not change over time.

\section{Adult Maternal Behaviors}

Latencies to exhibit adult maternal behavior. To determine the effects of early and juvenile experience on the initiation of maternal behavior, the latency to become maternal and the latency to display observed behaviors were analyzed with a series of 3 (early experience) $\times 2$ (juvenile experience) ANOVAs. For the latency to become maternal, there was a main effect of early experience $[F(2,37)=15.987, p<.004$; Figure 1]. There was also a main effect of early experience on latency to retrieve foster pups $[F(2,37)=6.251, p<.000$; Figure $2 \mathrm{~A}]$ and to lick foster pups $[F(2,37)=4.760, p<.014$; Figure $2 \mathrm{~B}$ ]. Short-separated, handled animals had shorter latencies to initiate these behaviors than did long-separated animals. Neither group differed from the undisturbed group. There were no other effects of early experience for other maternal behaviors or nonmaternal behaviors.

With respect to juvenile experience effects, there was a main effect of juvenile experience on the latency to lick foster pups $[F(1,37)=4.249, p<.046$; Figure $2 \mathrm{~B}]$ and to crouch over foster pups $[F(1,37)=10.214, p<.003$; Figure $2 \mathrm{C}]$. Animals that were exposed to pups during the juvenile period had longer (not shorter) latencies to initiate these behaviors than did animals that were not exposed to pups during the juvenile period. There were no other main effects for the juvenile experience. There were also no significant interactions between early experience and juvenile experience for latencies to exhibit any of the behaviors.

Quality of maternal responsiveness. To assess the effects of early and juvenile pup experience on the qual- ity of behavior that animals exhibited once animals became maternal (and had exhibited both retrieval and crouching), behaviors were analyzed for maternal animals on the final (5th) day of testing. There was a main effect of early experience for licking behavior $[F(2,26)=$ $5.209, p<.013$ ], with undisturbed animals showing more licking than short-separated animals (Figure 3B). There were no differences between long-separated groups and the other groups.

There was a main effect of juvenile experience for licking $[F(1,26)=12.56, p<.002$; Figure 3B]. Animals with juvenile exposure to foster pups (including both animals that did and those that did not retrieve as juveniles) exhibited less pup licking as adults than did animals without juvenile exposure to foster pups. There was also a significant early experience $\times$ juvenile experience interaction for retrieving $[F(2,26)=5.969, p<.007$; Figure $3 \mathrm{~A}]$ with undisturbed animals without juvenile experience spending more time retrieving pups than did undisturbed animals or short-separated animals with juvenile experience. There were no differences between long-separated animals and the other groups. There were no significant effects for other maternal or nonmaternal behaviors. Being exposed to pups during the juvenile period reduced the quality of behavior in adult animals on the last day of testing.

\section{Correlations Between Juvenile Behavior and Adult Behavior}

To determine whether there were individual differences in overall responsiveness to pups during adulthood, independent of previous early experience, correlations were computed between the average behavior (averaged over 5 days) and last-day behaviors of juvenile animals (only those exposed to pups) and the average be-

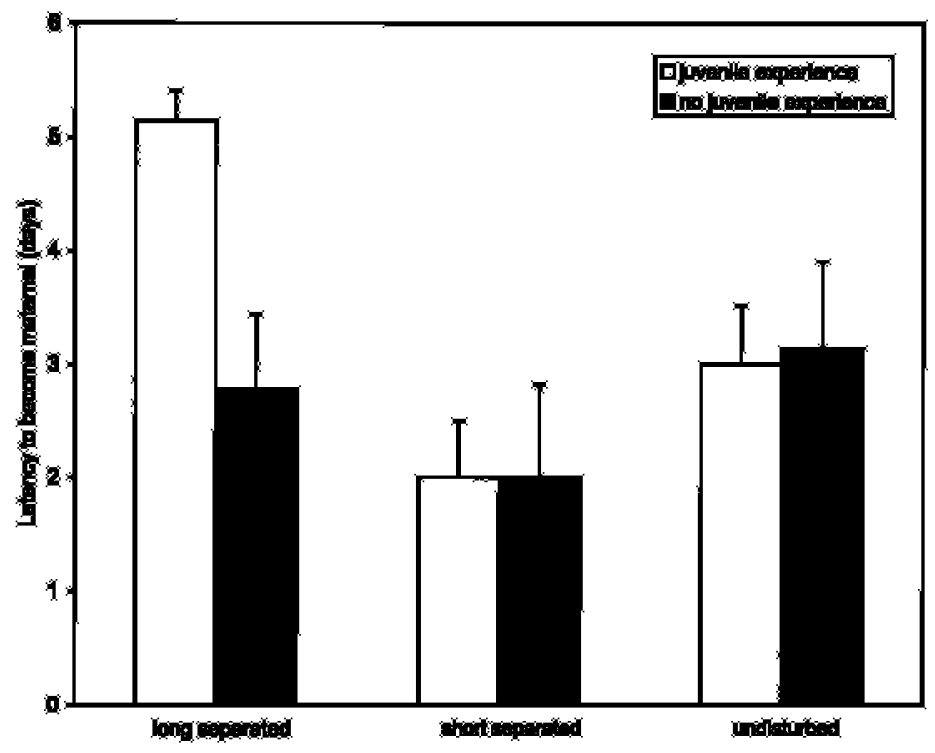

Figure 1. Latency to become maternal during adult maternal induction tests in Experiment 1 (mean, $S E M$ ). There was a significant main effect of early experience $[F(2,37)=15.99, p<.004]$. 

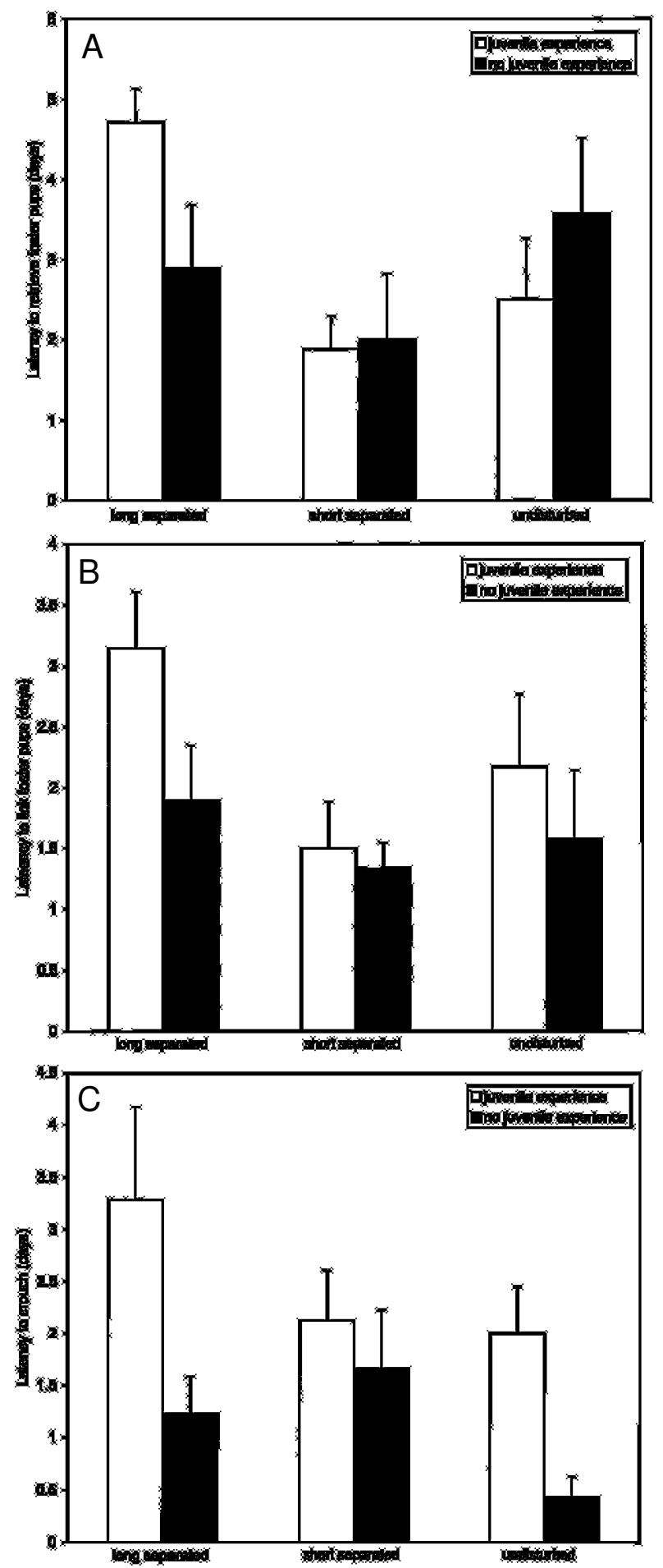

Figure 2. (A) Latency to retrieve foster pups during adult maternal induction tests in Experiment 1 (mean, $S E M)$. There was a significant main effect of early experience $[F(2,37)=6.25, p<$ $.000]$. (B) Latency to lick foster pups during adult maternal induction tests in Experiment 1 (mean, $S E M$ ). There were significant main effects of early experience $[F(2,37)=4.76, p<.014]$ and of juvenile experience $[F(1,37)=4.25, p<.046]$. (C) Latency to crouch over foster pups during adult maternal induction tests in Experiment 1 (mean, $S E M$ ). There was a significant main effect of juvenile experience $[F(1,37)=10.21, p<.003]$. havior and last-day behaviors of the same animals in adulthood $(n=21)$. All short-separated, long-separated, and undisturbed animals exposed to foster pups during the juvenile period and tested as adults were included in this analysis.

In general, juvenile retrieving behavior was related to adult maternal behaviors. Animals that retrieved more as juveniles spent more time retrieving $(r=.456, p<.038)$ and crouching $(r=.654, p<.001)$ as adults. When all the animals were included in the analysis, other juvenile behaviors were not related to adult maternal behaviors.

\section{Emergence Test and Open-Field Activity}

The emergence test and open-field activity were used to determine the effects of both early and juvenile experience on willingness to explore a novel environment and on emotional reactivity. Separate 3 (early experience) $\times$ 2 (juvenile experience) ANOVAs were computed for each emotionality measure. None of the emotionality or activity measures showed significant differences between early and/or juvenile experience groups.

\section{Correlations of Activity, Emergence Latencies} in the Open Field, and Adult Maternal Behavior

All the animals were assessed in terms of total activity, emergence latency, and proportion of time spent in the central area of the arena, and these measures were correlated with adult maternal behaviors on the final day of testing once the animals had become maternal, to assess whether differences in emotionality and fearfulness were reflected in the measures of adult maternal behavior. Separate analyses were then performed for each of the early experience groups. Significant correlations were found only for the long-separated animals. Significant correlations were found between activity scores, emergence latencies, and adult maternal behavior. Long-separated animals that were more active had shorter latencies to emerge from the start box $(r=-.720, p<.001)$. Longseparated animals that were also more active in the open field spent more time licking $(r=.757, p<.001)$ and had shorter latencies to become maternal $(r=-.629$, $p<.009)$. In addition, long-separated animals that emerged more rapidly into the open field (less "fearful") also licked foster pups more when maternal $(r=-.535$, $p<.033)$.

\section{Conclusion}

This experiment was designed to determine whether periods of separation from the mother during the first week of life would have a negative impact on later maternal behavior and whether exposure to pups during the juvenile period, when animals are quite responsive to pups, would enhance later maternal behavior. It was predicted that the juvenile experience with pups would reverse any deleterious effects of early maternal deprivation. The results demonstrated that although there were no effects (neither negative nor positive) of early separation on the response of juveniles to offspring, there were indeed effects of brief periods of separation, or handling, 

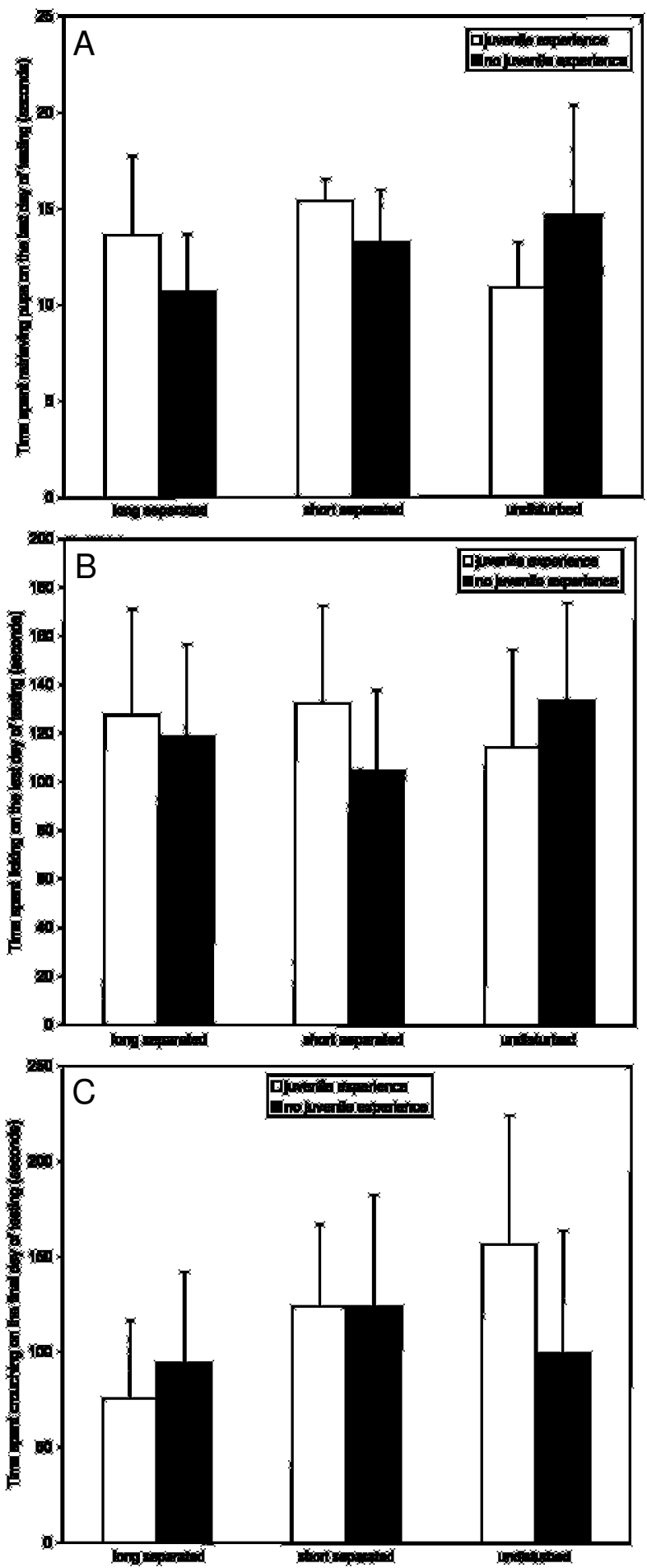

Figure 3. (A) Time spent retrieving foster pups on the final day of the adult maternal induction tests in Experiment 1 (mean, $S E M)$. There was a significant early experience $\times$ juvenile experience interaction $[F(2,26)=5.97, p<.007]$. (B) Time spent licking foster pups on the final day of the adult maternal induction tests in Experiment 1 (mean, $S E M$ ). There were main effects of early experience $[F(2,26)=5.21, p<.013]$ and of juvenile experience $[F(1,26)=12.56, p<.002]$. (C) Time spent crouching over foster pups on the final day of the adult maternal induction tests in Experiment 1 (mean, SEM). on later postpartum maternal behavior. In comparison with long-separated animals, the short-separated (handled) groups responded to pups more quickly and engaged in more pup licking, crouching, and retrieving during adulthood. It was also found that animals that had been exposed to foster pups as juveniles responded less quickly to pups than did animals that, as juveniles, were not exposed to foster pups, when both groups were tested as adults. Animals with juvenile exposure to foster pups also showed less maternal responsiveness, in terms of time spent licking pups, during the adult maternal tests. Our prediction that long periods of separation would cause deficits in adult maternal behavior and that these deficits would be reversed by juvenile experience with pups was incorrect. Long periods of separation had no effect on adult maternal behavior, whereas juvenile experience with pups had negative effects on the adult maternal behavior of long-separated animals.

\section{EXPERIMENT 2}

One problem for the interpretation of the first experiment lies in the fact that very few juveniles became maternal over the 5 days of juvenile experience with foster pups (approximately 10\%-30\%), so actual "maternal" experience was not displayed during the period. The reason the juvenile exposure to pups did not enhance adult maternal behavior may lie in the fact that the juvenile animals did not react maternally toward foster pups and, thus, did not gain a "maternal" experience. To more clearly establish the role of juvenile maternal experience and its interaction with early neonatal experiences on adult behavior, a second experiment was conducted. In this experiment, the amount of juvenile experience with foster pups was varied to include 8 days of exposure, 4 days of exposure, and no days of exposure. On the basis of pilot data, it has been found that $10 \%$ of juvenile animals given 4 days of exposure to foster pups will retrieve, whereas $90 \%$ of animals given 8 days of exposure to foster pups will retrieve.

Another difference between Experiment 1 and Experiment 2 is that in Experiment 1, the animals were raised after Day 30 singly in cages; hence, they were socially isolated from Day 30 to adulthood, although they were maintained in a room with many other rats and, therefore, had distal exposure to vocalizations and to odors of conspecifics. To ensure that any effects found in Experiment 1 were not due to confounds produced by isolation after the juvenile period, in this second experiment all the animals were raised in pairs and, hence, never experienced postpubertal isolation. Finally, in this second experiment, the early neonatal experience conditions included only the undisturbed and long-separation conditions; the short-separation (handling) condition was excluded. In this second experiment, we predicted that a long period of juvenile experience with foster pups would enhance the adult postpartum maternal behavior of undisturbed animals, whereas, as in Experiment 1, the group receiving short exposure to pups would show deficits in adult maternal behavior. 


\section{Method}

Subjects and Treatment

The subjects were 47 female Sprague-Dawley rats born at University of Toronto at Mississauga, from a stock originally obtained from Charles River Farms in Quebec. The treatment groups included the following: long separation/ 8 days of juvenile experience (LS/8JE; $n=8$ ); long separation/4 days of juvenile experience (LS/4JE; $n=7$ ); long separation/no juvenile experience (LS/NJE; $n=8$ ); undisturbed /8 days of juvenile experience (U/8JE; $n=7$ ); undisturbed /4 days of juvenile experience (U/4JE; $n=8$ ); undisturbed/no juvenile experience (U/NJE; $n=9$ ). The subjects were separated or left undisturbed from PNDs 2 to 9 . The same procedure was followed for long separation as that in Experiment 1.

\section{Maternal Behavior Test During the Juvenile Period}

The same procedure was followed as that in Experiment 1, except that juvenile animals were exposed to foster pups either continuously for 4 or 8 days or not at all. Following juvenile testing, each animal was housed with an untreated cohort until mating. There were 3 animals that did not mate after two mating attempts.

\section{Maternal Behavior Test During Adulthood}

Once again, during parturition, pups were removed from the subjects at 15-min intervals until the entire litter was born. After being isolated for 4 days following parturition, the maternal behavior for every animal was observed with the same procedure as that used in Experiment 1. Only 11 animals did not respond maternally.

\section{Results \\ Juvenile Maternal Behaviors}

Percent of juvenile animals showing maternal behaviors. As can be seen in Table 2, the group showing the highest percentage of animals that retrieved pups were undisturbed animals exposed to pups for 8 days $(80 \%)$. Separated animals with 8 days of exposure to pups showed the lowest percentage $(25 \%)$ of retrieval during the juvenile period. This difference among the four groups for percentage of retrieving was significantly different (chi-square, $p<.031$ ). This suggests that undisturbed animals with 8 days of exposure to rat pups gained a different experience than did separated animals with 8 days of exposure to rat pups. For the most part, the percentage of animals showing each of the other maternallike behaviors-licking, crouching, mouthing, and nestbuilding - did not differ among groups and was quite high for all (generally, between $80 \%$ and $100 \%$ of the animals). The only behavior that really differentiated the groups was retrieval behavior (Table 2).

Duration of maternal behaviors across the test period. Since in Experiment 1, there are clear changes across time in some responses of juveniles to pups in the juve-

Table 2

Percentage of Juvenile Animals Showing Maternal Behaviors in Experiment 2

\begin{tabular}{lccccc}
\hline & \multicolumn{2}{c}{ Separated Group } & & \multicolumn{2}{c}{ Undisturbed Group } \\
\cline { 2 - 3 } \cline { 5 - 6 } Behavior & 4 Days & 8 Days & & 4 Days & 8 Days \\
\hline Retrieve & 42 & 25 & & 40 & 80 \\
Body lick & 100 & 100 & & 100 & 100 \\
Genital lick & 100 & 100 & & 100 & 90 \\
Hover & 100 & 100 & & 100 & 100 \\
\hline
\end{tabular}

nile pup-experienced group, analyses were undertaken in which the first 4 days of testing in both juvenile experience groups (4- and 8-day groups) were included as a repeated measures factor. A series of 2 (early experience) $\times 4$ (days) repeated measures ANOVAs were computed on duration of all behaviors.

Consistent with Experiment 1, the animals demonstrated significant increases in time spent licking $[F(1,42)=$ $9.63, p<.003]$ and crouching $[F(1,42)=10.76, p<.002]$; in addition, these animals showed significant time effects for retrieving $[F(1,42)=4.71, p<.036]$ and sniffing pups $[F(1,42)=4.14, p<.048]$. In general, these changes reflected the fact that an increasing number of animals were becoming maternal over time. There were also significant day $\times$ early experience interactions for retrieving $[F(1,42)=5.08, p<.030]$ and licking $[F(1,42)=$ $7.81, p<.008]$. In both cases, the undisturbed group showed higher levels of these behaviors earlier in the test period than did the separated animals.

\section{Adult Maternal Behavior}

Latencies to exhibit behavior. To determine the effects of early and juvenile experience on the initiation of maternal behavior, the latency to become maternal and the latency to display observed behaviors were analyzed with a series of 2 (early experience) $\times 3$ (juvenile experience) ANOVAs. Similar to Experiment 1, there were no significant differences between long-separated and undisturbed groups in the latency to display any of the maternal and/or nonmaternal behaviors. There was a main effect of juvenile experience on the latency to crouch $[F(2,41)=3.79, p<.031 ;$ Figure $5 \mathrm{C}]$, with animals with 4 days of pup exposure during the juvenile period having longer latencies to crouch than did animals with no days of pup exposure during the juvenile period. The longer latencies to exhibit maternal behaviors seen in the juvenile pup group was consistent with the results of Experiment 1.

Quality of maternal responsiveness. To assess the effects of early and juvenile pup experience on the quality of behavior that animals exhibited when all the animals became maternal, behaviors were analyzed for maternal animals on the final day (5th) of testing, using 2 (early experience) $\times 3$ (juvenile experience) ANOVAs. There were no significant effects of early experience, juvenile experience, or interactive differences among groups for the quality of maternal responsiveness shown by adult maternal animals. These results are again consistent with Experiment 1, where differences that were found involved the short-separated handled groups and where the longseparated groups did not differ from the other groups.

\section{Correlations Between Juvenile Behavior and Adult Behavior}

To determine whether there were individual differences in overall responsiveness to pups during adulthood that related to their responsiveness as juveniles (independent of previous early experience), correlations were computed to compare average behavior across days and 
behavior on the last day of testing shown by animals exposed to foster pups as juveniles to the duration of these same behaviors in adulthood $(n=30)$. All the animals in the 4-day and 8-day juvenile groups were included.

Consistent with Experiment 1, average juvenile retrieving was positively related to the average of adult animals' mouthing and pup pick-ups $(r=.557, p<.001)$, licking $(r=.349, p=.059)$, and digging $(r=.417, p<$ .022 ). Moreover, in contrast to Experiment 1 , in this study, where a higher proportion of juvenile animals showed pup retrieval than in Experiment 1, other juvenile maternal behaviors were also associated with adult maternal behaviors. For example, juvenile licking was positively related to adult licking $(r=.540, p<.002)$ and adult crouching $(r=.431, p<.017)$. Also, juvenile crouching was positively related to adult licking $(r=.397, p<.030)$, and average juvenile mouthing was positively related to adult licking $(r=.515, p<.004)$, crouching $(r=.395$, $p<.031)$, mouthing $(r=.369, p<.045)$, and nest building $(r=.533, p<.002)$.

\section{Adult Maternal Behavior:}

\section{Animals That Retrieved as Juveniles}

The observation in Experiment 1 that juvenile retrievals were positively associated with adult maternal behaviors could suggest either that the experience of retrieval enhances later responsiveness as an adult or that it reflects a responsiveness trait. To investigate the causal hypothesis, the 8-day juvenile group was included, in which a high proportion of animals come to retrieve during the juvenile period. Since some animals exposed for 4 days also retrieve, the next analyses were undertaken to compare adult maternal behavior in animals that retrieved as juveniles with those that did not and with those that were simply exposed to same-age conspecifics. Additional 2 (early experience) $\times 3$ (retrieving condition: with pup exposure and retrieved, with pup exposure and not retrieved, and not with pup) ANOVAs were performed on latencies to exhibit behaviors and on the quality of the maternal response once the animals became maternal.

Latencies. There were no significant effects on the latencies to exhibit maternal behaviors (Figures 4 and 5).

Quality of maternal responsiveness. When all the groups were compared, there were no main effects of either early experience or juvenile retrieving on the quality of maternal responsiveness on the last day of adult maternal testing. There was, however, a significant early experience $\times$ juvenile retrieving interaction on the quality of licking on the last day of testing $[F(2,41)=5.364, p<$ .009], with separated animals showing the most licking when they had retrieved as juveniles and undisturbed animals showing the most licking when they received no exposure to pups during the juvenile period (Figure 6).

\section{Conclusion}

This experiment was designed to determine whether long periods of juvenile exposure to pups (8 days) would result in high levels of retrieving, as compared with short periods of exposure to pups (4 days), so that the influence of retrieving as a juvenile could be investigated in the adult postpartum maternal behavior. Also, the second experiment was designed to compensate for any effects of postjuvenile isolation found in the first experiment by rearing the animals in pairs from the juvenile tests to adulthood.

In this second experiment, there were no effects of early separation on adult maternal behavior, which was not surprising, because the main effects of early experi-

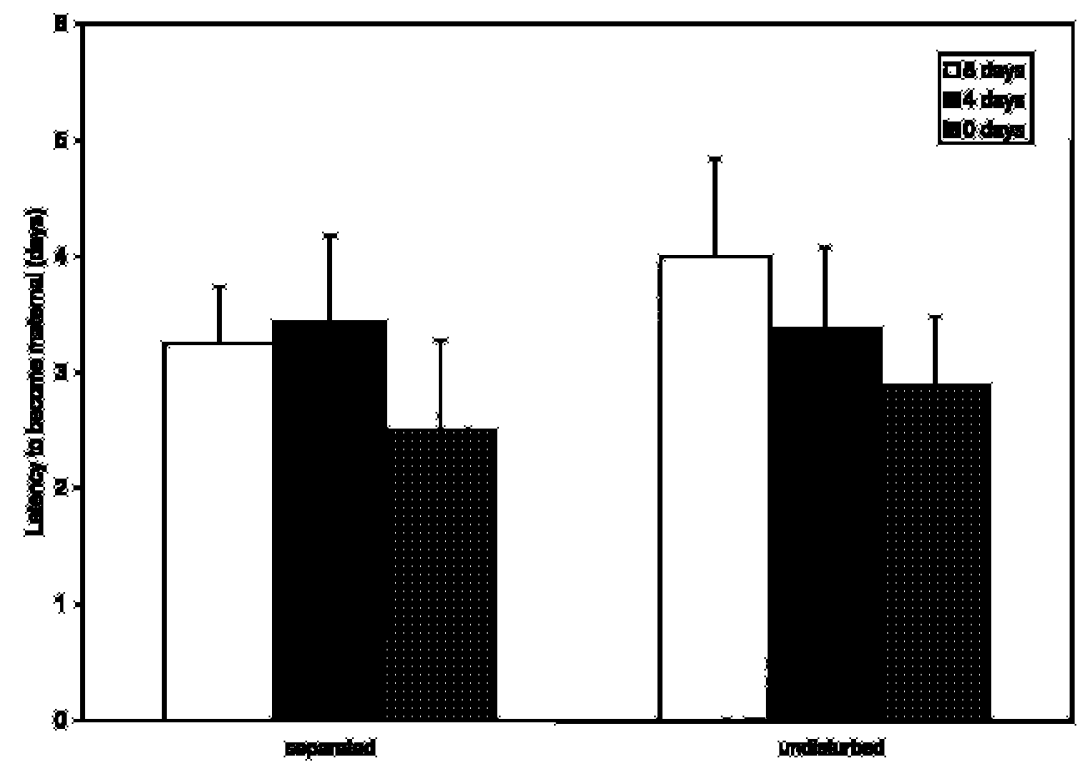

Figure 4. Latency to become maternal during adult maternal induction tests in Experiment 2 (mean, $S E M$ ). 

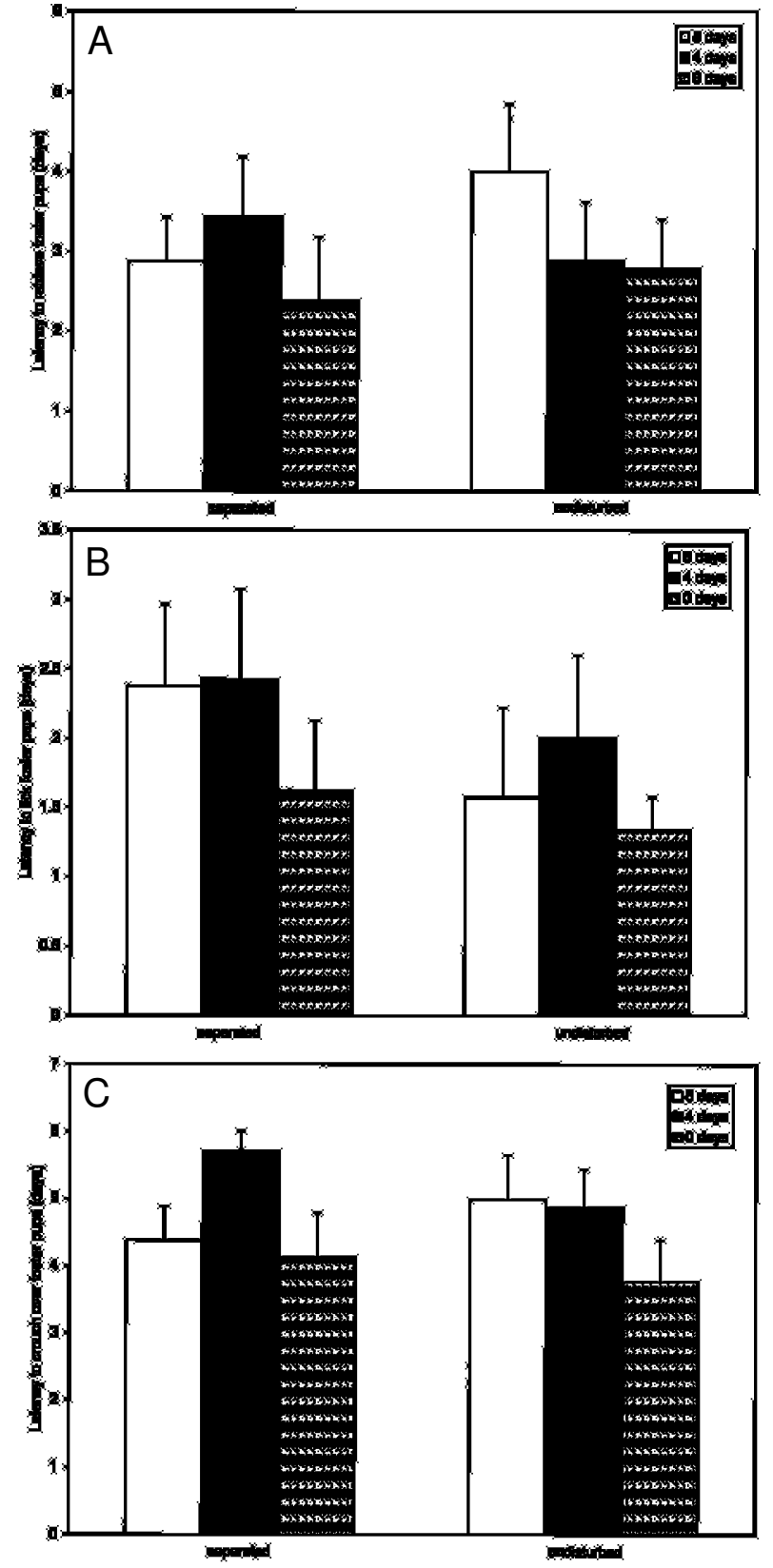

Figure 5. (A) Latency to retrieve foster pups during adult maternal induction tests in Experiment 2 (mean, SEM ). (B) Latency to lick foster pups during adult maternal induction tests in Experiment 2 (mean, $S E M$ ). (C) Latency to crouch over foster pups during adult maternal induction tests in Experiment 2 (mean, $S E M)$. There was a main effect of juvenile experience $[F(2,41)=$ $3.79, p<.031]$.

ence were only found when the short-separated handled group was included. During the juvenile period, there were differences between early experience groups in the proportion of animals that were exposed to pups for 8 days that retrieved pups. The separated animals showed significantly less retrieving than did the undisturbed animals in this 8-day group. This difference during the juvenile period, however, did not carry over into adulthood or produce differences in adulthood. There were also effects of juvenile experience on adult maternal behavior, with animals that received 4 days of pup exposure during the juvenile period showing decreased maternal responsiveness. Long periods ( 8 days) of juvenile exposure to pups had neither positive nor negative effects on adult maternal behavior.
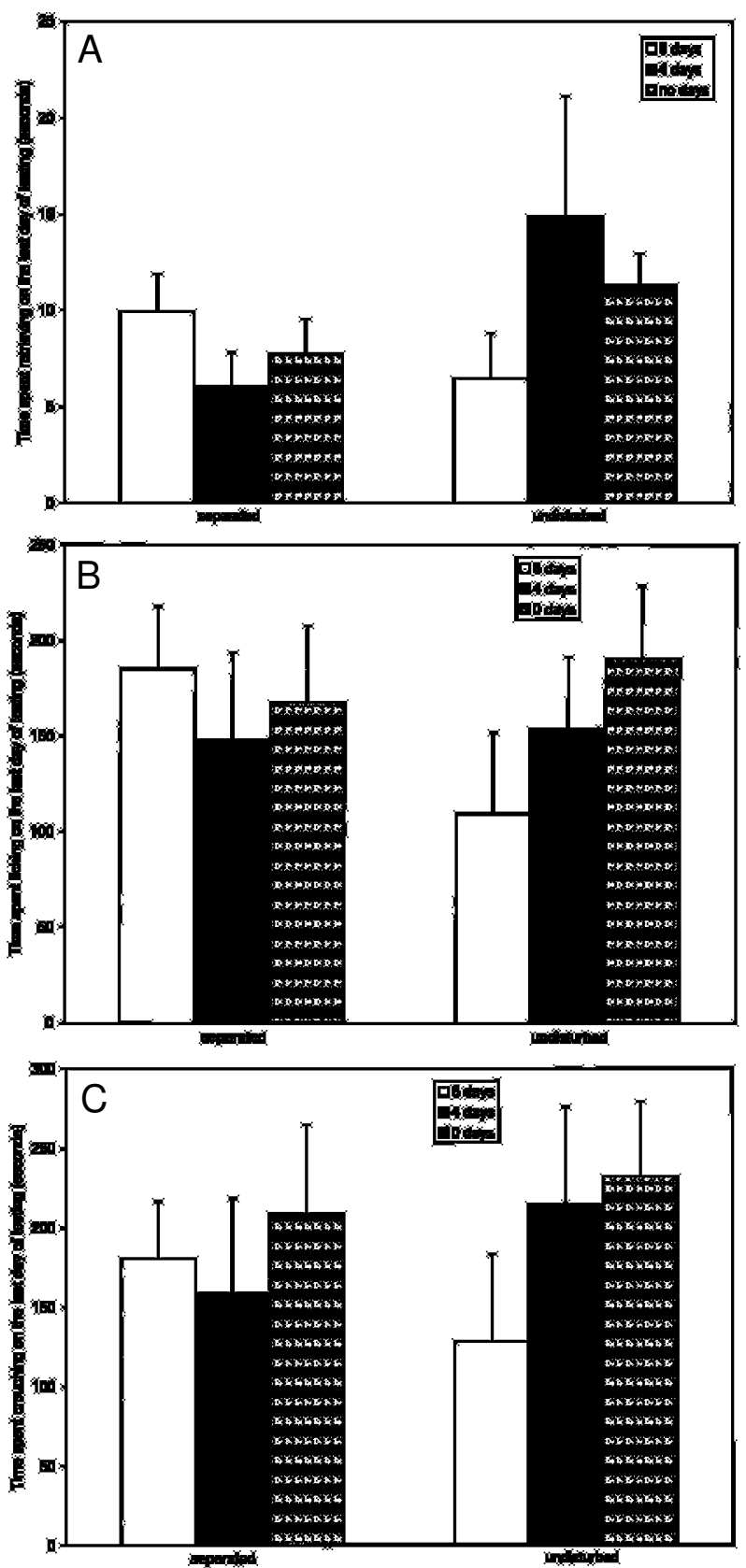

Figure 6. (A) Time spent retrieving foster pups on the final day of the adult maternal induction tests in Experiment 2 (mean, $S E M)$. (B) Time spent licking foster pups on the final day of the adult maternal induction tests in Experiment 2 (mean, $S E M$ ). (C) Time spent crouching over foster pups on the final day of the adult maternal induction tests in Experiment 2 (mean, $S E M$ ). 


\section{GENERAL DISCUSSION}

When animals are separated from their mothers and littermates, they experience reduction or loss of exposure to maternal licking and nurturance and to warmth and contact comfort associated with the littermates. They also experience novel characteristics of the environment in which they are placed, including new smells and a changed somatosensory and thermal environment. Two experiments were designed to determine whether brief daily periods of separation from the mother and littermates during the 1 st week of life would have a negative impact on later maternal behavior and whether exposure to newborn foster pups during the juvenile period, when animals are quite responsive to pups, would enhance later maternal behavior. It was predicted that juvenile experiences interacting with pups would reverse any deleterious effects of early maternal separation. Overall, there were few negative effects of $3 \mathrm{~h}$ of daily maternal separation on later maternal behavior in either experiment, and in fact, very brief periods of separation (15 min daily, the so-called handling regime) in Experiment 1 actually enhanced adult maternal behavior. The juvenile exposure to pups had negative, rather than positive, effects on adult maternal behavior.

In Experiment 1, a closer analysis of the groups separated for $3 \mathrm{~h}$ daily indicated that early separation had adverse effects on later behavior only if the separated animals received an intervening juvenile experience with pups; during that experience, they crouched with and licked pups but did not show the full juvenile maternal pattern normally associated with pup retrieval. Rather than reversing the negative effects of early deprivation, and unlike previous research that has shown that juvenile experience with pups enhances later maternal behavior (Gray \& Chesley, 1984), pup exposure in juveniles produced negative effects. Potentially, these negative effects may be due to some interaction of juvenile experience with parturitional hormones in adulthood, because other evidence suggesting that juvenile maternal experience is beneficial was demonstrated in adult virgin rats (Gray \& Chesley, 1984), whereas our evidence, suggesting that juvenile experience is negative, was found in adult postpartum rats.

In our experiment, separated postpartum animals with juvenile experience took longer to lick and hover over foster pups in adulthood and showed less retrieving during the earlier part of the testing period, when compared with separated animals without juvenile experience. Interestingly, such negative effects of juvenile pup experience on maternal behaviors were not found among either the undisturbed animals or the short-separated handled animals. This pattern of results was reflected in a significant interaction between early deprivation and juvenile experience on adult maternal behaviors. These data suggest that the early separation experience somehow sensi- tizes the animal to the aversive properties of novel stimuli (pups) and that the negative effects of these stimuli are then enduring.

Although these data suggest that juvenile experience with pups, which usually includes extensive pup licking and hovering over pups, may in fact be deleterious to adult maternal behaviors, we cannot conclude on the basis of these results that the experience of interacting with pups maternally would have no beneficial effects. In studies of adult animals, full maternal behavior is almost invariably associated with pup retrieval. In an attempt to investigate the effects of juvenile pup retrieval on adult maternal behavior, we, therefore, included in Experiment 2 a group of animals that, as juveniles, received either 4 or 8 days of exposure to pups (rather than the 5 days of experience included in the first experiment). These groups were included in the hope that juvenile animals would be sensitized to pups and show retrieving after the 8 days and that juvenile animals with 4 days experience would merely be exposed to pup cues and would engage in social licking and crouching but would not show any retrieving behavior.

The results showed that juveniles exposed to pups for 8 days exhibited high levels of retrieving during the juvenile period if they had not previously been separated from their mothers, but low levels of juvenile retrieval behavior if they had been separated from their mothers. These results suggest that early deprivation from the mother does affect how juveniles respond to pups. However, in adulthood where there were no effects of early preweaning separation, it was found that short periods of pup exposure (4 days) during the juvenile period had a negative effect on adult maternal behavior (as indicated earlier), whereas long periods of pup exposure during the juvenile period ( 8 days) had neither positive nor negative effects.

\section{Effects of Early Separation}

The absence of strong long-term main effects of early separations on adult maternal behavior in the present study was surprising in light of the considerable physiological changes that occur with these daily separations (Kuhn \& Schanberg, 1998) and the observations of clear deficits with other separation regimes. For instance, we have found that 5-h daily separations, and 24-h daily separations over the first 18 days of life have very clear negative effects on adult maternal behavior (A. Gonzalez et al., 2001; Lovic et al., 2001). It may well be that the extent of daily 3 -h separations over the 1 st week of life in the present study was simply too short and over too brief a period to produce long-term negative effects into adulthood, although they clearly had short-term effects in the juvenile period.

Given the extensive retarding effects of this separation regime on cell multiplication, growth, and development, neuroendocrine function, and cellular responses to the 
major trophic hormones, the relative intactness of maternal behavior is quite striking and speaks to the resilience of the organism (Kuhn \& Schanberg, 1998).

\section{Effects of Short Periods of Separation}

The finding that early handling, or very brief periods of daily separation, had beneficial effects on adult maternal behavior indicates that manipulations during this period can have long-term and enduring effects within the maternal behavior context. Although we do not know precisely how these early handling effects affect maternal behavior, there is evidence that these manipulations probably result in an enhancement of licking by mothers when pups are returned to the nest that persists into the next day (M. Gonzalez, Ladd, Huot, Owents, \& Plotsky, 1999; Pryce, Bettschen, \& Feldon, 2001). When animals experiencing long separations are returned to the nest, they also may experience a brief period of heightened licking on return to the litter; however, in this case, the overall licking and maternal attention received across the day does not differ from those for controls (Pryce et al., 2001). The fact that they have access to the mother for $3 \mathrm{~h}$ less in a $24-\mathrm{h}$ period would suggest, however, that they are receiving considerably less maternal attention than are animals that remain with their mothers throughout. The short-separation, early handling experience not only affects adult maternal behavior, but also has the effect of reducing fear-mediated behaviors (Caldji et al., 2000) and behavioral responsiveness to stressors and of enhancing learning as well (Meaney, Aitken, Bhatnagar, \& Sapolsky, 1991; Meaney, Aitken, van Berkel, Bhatnagar, \& Sapolsky, 1988; Vallee et al., 1999). All these behavioral effects of early handling may well increase an animal's willingness to interact with pups. The role of altered endocrinology in the behavioral effects are not at present clear, however, with some investigators finding an effect of early handling on open-field and other "emotion" tests (Caldji et al., 2000; Caldji et al., 1998; Meerlo, Horvath, Nagy, Bohus, \& Koolhaas, 1999) and others finding no effect (Campbell \& Spear, 1999).

\section{Early Separation and the HPA Axis}

There is a substantial literature that shows that early maternal separation produces an altered HPA stress response in adulthood (Plotsky \& Meaney, 1993), an effect that very likely develops during the early neonatal stages (McCormick, Kehoe, \& Kovacs, 1998). Short periods of maternal separation, handling, causes lowered corticosterone response to stressors, decreased sensitivity to $\mathrm{ACTH}$, and decreased CRF depletion in response to stress, elevated levels of negative-feedback hippocampal GC receptors, as well as other changes that create an animal with decreased HPA responsiveness and a shortened stress response (Liu et al., 1997; Plotsky \& Meaney, 1993; Suchecki, Nelson, van Oers, \& Levine, 1995). Although there is evidence that the HPA system may be involved in behavioral expressions of fear, this is not consistently shown in our study. Moreover, how these HPA changes would translate into altered maternal behavior is not known. Recent work by Panesar, Rees, and Fleming (2000), in fact, indicates that higher levels of corticosterone (in drinking water of adrenalectomized postpartum animals) enhances, rather than depresses, the expression of their maternal behavior. These same doses in the virgin have no effect on latency to respond maternally. Whatever the effects of the HPA axis might be in relation to maternal behavior, it is certainly unlikely to be a direct one.

\section{Early Separation and the Dopamine System}

Early stimulation and stress in the nest not only alters the development of the HPA axis, but also affects the mesolimbic dopamine (DA) system (Hall, Wilkinson, Humby, \& Robbins, 1999; Jaskiw, Karoum, \& Weinberger, 1990). These effects could, in turn, alter learning and reinforcement mechanisms. The normal regulation of the mesolimbic dopamine system is altered by early life experience and is involved in maternal behavior (Hansen, 1994; Hansen, Bergvall, \& Nyiredi, 1993). DAs disrupt maternal retrievals and the animal's motivation to respond maternally (Keer \& Stern, 1999). They also reduce the reinforcing properties of rat pups (Fleming, Korsmit, \& Deller, 1994). Although current evidence suggests that only the mesolimbic dopamine system is affected by early experiences, it would be interesting to establish whether early experience also affects the hypothalamic dopamine system. Changes to the hypothalamic dopamine system may also affect the prolactin, since dopamine is a prolactin inhibitor (Kramer \& Hopkins, 1982) and prolactin is an important hormone mediating the expression of maternal behavior (Bridges, 1994). Prolactin has also been related to the expression of maternal responsiveness during the juvenile period (Kinsley \& Bridges, 1988). This suggests that the effects of early deprivation on the dopamine system could possibly affect how the prolactin system develops and functions during the juvenile period, which may lead to subsequent changes in adult prolactin function and maternal behavior.

\section{Juvenile Maternal Behavior}

What are the behavioral and physiological mechanisms of the effects of juvenile experience on adult maternal behavior? One possible explanation is that novel pup cues in the juvenile period may be particularly stressful. If these novel cues are encountered during a biologically irrelevant time (and when hormones are not present), a long-term aversion or reduction in the positive valence or reinforcing properties of pups could be produced. Alternatively, it is possible that, for the juvenile animal, pups are initially processed differently or maternal experiences are less well consolidated. Unfortunately, on the basis of the behavioral results from the juvenile period, there is no evidence that juvenile animals in the early deprived groups were particularly stressed, that they responded with heightened withdrawal or aversion to pups, or that they treated pups at all 
differently. In fact, most juveniles, when placed with pups, stayed in close proximity to them, licked them, and hovered over them. However, the observations taken during the juvenile period may not have been adequately sensitive to detect subtle avoidance behaviors.

An explanation of the absence of a positive juvenile effect on adult maternal behavior in undisturbed animals may be one of state dependency. Pups may not be perceived in the same way if they are encountered under conditions of hormonal stimulation than when they are not. In fact, as juveniles, animals may not perceive pups as maternally relevant at all, and consequently, the behavior they exhibit in the presence of pups may reflect nonspecific social, or play, behavior toward pups, rather than maternal behavior (Brunelli et al., 1985). Others have found that exposure of juvenile animals to pups does not result in complete and consistent maternal behavior (Brunelli et al., 1985), although the sensitization of nulliparous adult animals with pups, adult animals that may find pups biologically irrelevant owing to the lack of hormonal influence, does result in comprehensive maternal interactions (Fleming \& Rosenblatt, 1974). This suggests that maternal behavior may be somehow systematically different for animals at different developmental stages. Because postpartum animals are immediately responsive to pups following birth and nulliparous and juvenile animals do not show immediate maternal responsiveness to foster pups (Fleming et al., 1999; Fleming \& Rosenblatt, 1974), maternal behavior for animals under hormonal stimulation may be systematically different than that for animals without hormonal stimulation.

\section{Neuroanatomy of Juvenile Maternal Behavior}

In contrast to our results, Gray and Chesley (1984) found a positive effect of juvenile maternal behavior (and retrieval) on the sensitization of maternal behavior in virgin rats, although the juvenile exposure period was longer at 10 days of exposure. Since our groups were tested during the postpartum period, however, the effects of fluctuating postpartum hormone levels may also interrupt the enhancement of adult maternal behavior by the juvenile maternal experience. That the juvenile experience has some effect is shown by the fact that the 4-day exposure group showed negative effects on adult behavior, whereas the 8-day group did not. Although maternal behavior during the juvenile period resembles maternal behavior during adulthood, there are some differences that seem to suggest that different systems may be at work (Brunelli et al., 1985; Gray \& Chesley, 1984). For example, it has been demonstrated that only the lateral habenula displays greater c-Fos expression in maternal juveniles, when compared with nonmaternal juveniles.

Other areas, such as the lateral habenula, the medial preoptic area, and the ventral bed nucleus of the stria terminalis, differed in c-Fos expression between maternal and nonmaternal adult animals, but not in juvenile animals (Drmic, Oxley, \& Fleming, 2001; Kalinichev, Rosenblatt, Nakabeppu, \& Morrell, 2000). This seems to suggest that although the lateral habenula and the bed nucleus are involved in juvenile and adult maternal behavior, other areas may not be mature enough for involvement in maternal behavior during the juvenile period. For example, small lesions to the MPOA of juvenile rats will not disrupt maternal behavior during the postweaning period; however, large lesions will (Kalinichev, Rosenblatt, \& Morrell, 2000), which suggests that the MPOA may not be fully developed in the juvenile animal. The different systems used for juvenile maternal responsiveness and adult maternal responsiveness may account for why separated animals do not differ from controls during the juvenile period but do differ during adulthood, since early maternal deprivation may alter the development of adult maternal systems.

\section{Conclusion}

In conclusion, $3 \mathrm{~h}$ of daily separations from the mother and the nest do not have long-term negative effects on later maternal behavior, whereas brief separations associated with the handling regime enhance later behavior. Exposure to pups for short periods during the juvenile period also has powerful negative effects on subsequent maternal behavior, especially in animals that were previously maternally deprived, as was seen in Experiment 1. Longer periods of exposure to pups during the juvenile period produces neither positive nor negative effects on adult postpartum maternal behavior. The ecological relevance of these findings is not clear. Rattus norvegicus is a species in which alloparenting or paternal behavior does not occur and in which juveniles are unlikely to be called upon to participate in caretaking. It would be interesting to know whether similar negative effects would occur in species in which adult behavior is heavily dependent on earlier experiences during the juvenile and subadult periods, as in marmoset monkeys and even humans.

\section{REFERENCES}

Anisman, H., Zaharia, M. D., Meaney, M. J., \& Merali, Z (1998). Do early-life events permanently alter behavioral and hormonal responses to stressors? International Journal of Developmental Neuroscience, 16, 149-164.

BRIDGES, R. S. (1994). The role of lactogenic hormones in maternal behavior in female rats. Acta Paediatrica, 397 (Suppl.), 33-39.

Brunelli, S. A., Shindledecker, R. D., \& Hofer, M. A. (1985). Development of maternal behavior in prepubertal rats at three ages: Age characteristic patterns of responses. Developmental Psychobiology, 18, 309-326.

Caldji, C., Francis, D., Sharma, S., Plotsky, P. M., \& Meaney, M. J. (2000). The effects of early rearing environment on the development of GABAA and central benzodiazepine receptor levels and noveltyinduced fearfulness in the rat. Neuropsychopharmacology, 22, 219-229.

Caldji, C., Tannenbaum, B., Sharma, S., Francis, D., Plotsky, P. M., \& MeAney, M. J. (1998). Maternal care during infancy regulates the development of neural systems mediating the expression of fearfulness in the rat. Proceedings of the National Academy of Sciences, 95, 5335-5340.

Campbell, J., \& Spear, L. P. (1999). Effects of early handling on amphetamine-induced locomotor activation and conditioned place preference in the adult rat. Psychopharmacology, 143, 183-189.

Dorner, G., Plaschke, M., Tonjes, R., \& Wenzel, J. (1984). Teratomorphogenic effects on the brain produced by neonatal maternal deprivation can be partly prevented by pyridosigmine administration. Experimental \& Clinical Endocrinology, 84, 352-355. 
Drmic, I., Oxley, G., \& Fleming, A. S. (2001). Changes in PKC associated with maternal behavior in juvenile and adultfemale rats. Manuscript submitted for publication.

Fleming, A. S., Cheung, U., Myhal, N., \& Kessler, Z (1989). Effects of maternal hormones on "timidity" and attraction to pup-related odors in female rats. Physiology \& Behavior, 46, 449-453.

Fleming, A. S., Korsmit, M., \& Deller, M. (1994). Rat pups are potent reinforcers to the maternal animal: Effects of experience, parity, hormones, and dopamine function. Psychobiology, 22, 44-53.

Fleming, A. S., Lovic, V., Gonzalez, A., Rees, S., Melo, A., \& Kraemer, G. (2001). Mothering begets mothering. Manuscript submitted for publication.

Fleming, A. S., \& LuebKe, C. (1981). Timidity prevents the virgin female rat from being a good mother: Emotionality differences between nulliparous and parturient females. Physiology \& Behavior, 27, 863-868.

Fleming, A. S., O'Day, D. H., \& Kraemer, G. W. (1999). Neurobiology of mother-infant interactions: Experience and central nervous system plasticity across development and generations. Neuroscience \& Biobehavioral Reviews, 23, 673-685.

Fleming, A. S., \& Rosenblatt, J. S. (1974). Maternal behavior in the virgin and lactating rat. Journal of Comparative \& Physiological Psychology, 86, 957-972.

Francis, D., Diorio, J., Liu, D., \& Meaney, M. J. (1999, November). Nongenomic transmission across generations of maternal behavior and stress response in the rat. Science, 286, 1155-1158.

GonZalez, A., \& Fleming, A. S. (2001). The effects of artificial rearing on juvenile behavior. Manuscript in preparation.

Gonzalez, A., Lovic, V., Ward, G. R., Wainwright, P. E., \& Fleming, A. S. (2001). Intergenerational effects of complete maternal deprivation and replacement stimulation on maternal behavior and emotionality in female rats. Developmental Psychobiology, 38, 11-32.

Gonzalez, M., Ladd, C. O., Huot, B. L, Owents, M. J., \& Plotsky, P. M. (1999). Prevention of HPA axis changes in response to neonatal maternal separation by providing dams with foster pups during the absence of her litter. Society for Neuroscience Abstracts, 25, 1455.

Gray, P., \& Chesley, S. (1984). Development of maternal behavior in nulliparous rats (Rattus norvegicus): Effects of sex and early maternal experience. Journal of Comparative Psychology, 98, 91-99.

Hall, F. S. (1998). Social deprivation of neonatal, adolescent, and adult rats has distinct neurochemical and behavioral consequences. Critical Reviews in Neurobiology, 12, 129-162.

Hall, F. S., Wilkinson, T. H., Humby, T., \& Robbins, T. W. (1999). Maternal deprivation of neonatal rats produces enduring changes in dopamine function. Synapse, 32, 37-43.

HANSEN, S. (1994). Maternal behavior of female rats with 6-OHDA lesions in the ventral striatum: Characterization of the pup retrieval deficit. Physiology \& Behavior, 55, 615-620.

Hansen, S., Bergvall, A. H., \& Nyiredi, S. (1993). Interaction with pups enhances dopamine release in the ventral striatum of maternal rats: A microdialysis study. Pharmacology, Biochemistry \& Behavior, 45, 673-676.

Jaskiw, G. E., Karoum, F. K., \& Weinberger, D. R. (1990). Persistent elevations in dopamine and its metabolites in the nucleus accumbens after mild subchronic stress in rats with ibotenic acid lesions of the medial prefrontal cortex. Brain Research, 534, 321-323.

Kalinichev, M., Rosenblatt, J. S., \& Morrell, J. I. (2000). The medial preoptic area, necessary for adult maternal behavior in rats, is only partially established as a component of the neural circuit that supports maternal behavior in juvenile rats. Behavioral Neuroscience, 114, 196-210.

Kalinichev, M., Rosenblatt, J. S., NaKabeppu, Y., \& Morrell, J. I. (2000). Induction of c-Fos- and FosB-like immunoreactivity forebrain neuronal populations differentially involved in pup-mediated maternal behavior in juvenile and adult rats. Journal of Comparative Neurology, 416, 45-78.

KeER, S. E., \& Stern, J. M. (1999). Dopamine receptor blockade in the nucleus accumbens inhibits maternal retrieval and licking, but en- hances nursing behavior in lactating rat. Physiology \& Behavior, 67, 659-669.

KInsLEy, C. H., \& BRIDges, R. S. (1988). Prolactin modulation of the maternal-like behavior displayed by juvenile rats. Hormones \& Behavior, 22, 49-65.

Kramer, I. M., \& Hopkins, C. R. (1982). Studies on the kinetics of dopamine-regulated prolactin secretion. Molecular \& Cellular Endocrinology, 28, 191-198.

Kuhn, C. M., \& Schanberg, S. M. (1998). Responses to maternal separation: Mechanisms and mediators. International Journal of Developmental Neuroscience, 16, 261-270.

Lehmann, J., Pryce, C. R., Bettschen, D., \& Feldon, J. (1999). The maternal separation paradigm and adult emotionality and cognition in male and female Wistar rats. Pharmacology, Biochemistry \& Behavior, 64, 705-715.

Liu, D., Diorio, J., Tannenbaum, B., Caldi, C., Francis, D., Freedman, A., Sharma, S., Pearson, D., Plotsky, P. M., \& Meaney, M. J. (1997, September). Maternal care, hippocampal glucocorticoid receptors, and hypothalamic-pituitary-adrenal responses to stress. Science, 277, 1659-1662.

Lovic, V., Gonzalez, A., \& Fleming, A. S. (2001). Maternally separated rats show deficits in maternal care in adulthood. Developmental Psychobiology, 39, 19-33.

Mayer, A. D., \& Rosenblatt, J. S. (1998). A method for regulating the duration of pregnancy and the time of parturition in Sprague-Dawley rats (Charles River CD strain). Developmental Psychobiology, 32,131136.

McCormick, C. M., Kehoe, P., \& Kovacs, S. (1998). Corticosterone release in response to repeated, short episodes of neonatal isolation: Evidence of sensitization. International Journal of Developmental Neuroscience, 16, 1-10.

Meaney, M. J., Aitken, D. H., Bhatnagar, S., \& Sapolsky, R. M. (1991). Postnatal handling attenuates certain neuroendocrine, anatomical, and cognitive dysfunctions associated with aging in female rats. Neurobiology of Aging, 12, 31-38.

Meaney, M. J., Aitken, D. H., van Berkel, C., Bhatnagar, S., \& SaPOLSKY, R. M. (1988). Effect of neonatal handling on age-related impairments associated with the hippocampus. Science, 239, 766-768.

Meerlo, P., Horvath, K. M., Nagy, G. M., Bohus, B., \& Koolhaas, J. M. (1999). The influence of postnatal handling on adult neuroendocrine and behavioral stress reactivity. Journal of Neuroendocrinology, 11, 925-933.

Panesar, S., Rees, S. L., \& Fleming, A. S. (2000). Effects of adrenalectomy and corticosterone replacement on maternal behavior in primiparous and virgin (SD) rats. Society for Neuroscience Abstracts, 26, 2036.

Plotsky, P. M., \& Meaney, M. J. (1993). Early, postnatal experience alters hypothalamic corticotropin-releasing factor (CRF) mRNA, median eminence CRF content and stress-induced release in adult rats. Molecular Brain Research, 18, 195-200.

Pryce, C. R., Bettschen, D., \& Feldon, J. (2001). Comparison of the effects of early handling and early deprivation on maternal care in the rat. Behavioral Neuroscience, 38, 239-251.

Suchecki, D., Nelson, D. Y., Van Oers, H., \& Levine, S. (1995). Activation and inhibition of the hypothalamic-pituitary-adrenal axis of the neonatal rat: Effects of maternal deprivation. Psychoneuroendocrinology, 20, 169-182.

Vallee, M., MacCari, S., Dellu, F., Simon, H., Le Moal, M., \& MAYO, W. (1999). Long-term effects of prenatal stress and postnatal handling on age-related glucocorticoid secretion and cognitive performance: A longitudinal study in the rat. European Journal of Neuroscience, 11, 2906-2916.

Wilson, D. A., \& Sullivan, R. M. (1994). Neurobiology of associative learning in the neonate: Early olfactory learning. Behavioral \& Neural Biology, 61, 1-18.

(Manuscript received June 15, 2000; revision accepted for publication April 24, 2001.) 